\title{
Ligadura interesfinteriana del trayecto fistuloso (LIFT) como alternativa de tratamiento a una fístula perianal compleja
}

\author{
Natalia Moreno B. ${ }^{1,2}$, Alejandro Readi V. ${ }^{2}$, Ernesto Melkonian T. ${ }^{2}$, \\ Rodrigo Capona P. ${ }^{2}$ y Mónica Martínez M. ${ }^{2}$
}

'Departamento de Cirugía Hospital Clínico Universidad de Chile. Santiago, Chile. Departamento de Cirugía Hospital del Salvador. Santiago, Chile

Recibido el 1 de octubre de 2017 y aceptado para publicación el 22 de mayo de 2018

Correspondencia a: Dra. Natalia Moreno B. anatalia96@gmail.com

\section{Ligation of intersphincteric fistula tract (LIFT) as an alternative treatment for complex perianal fistula.}

Introduction: Surgical techniques for complex perianal fistula have high recurrence and fecal incontinence rates. The technique of LIFT (ligation of the intersphincteric fistula tract) has achieved lower rates of recurrence with almost no risk of incontinence according to international reports. However, it is not yet consolidated as a standard technique for this pathology. Objective: To present the results (clinical success and incontinence according to the Wexner scale) of our patients with complex perianal fistula operated with the LIFT technique. Materials and Method: Descriptive study of a non-randomized prospective cohort, with patients operated for complex perianal fistula at the Hospital del Salvador, between 2015 and 2017. Results: 22 operated patients are included. In 77\%, therapeutic success is obtained in the first surgery and up to $90 \%$ with a second surgery. None of this patients modified their preoperative Wexner. Conclusions: In patients with complex perianal fistula, the LIFT technique is an alternative that offers high cure rates with low risk of fecal incontinence.

Key words: complex fistula; LIFT; fecal incontinence.

\section{Resumen}

Introducción: Las técnicas quirúrgicas para la fístula perianal compleja han tenido altas tasas de recidiva asociado al riesgo de incontinencia fecal. La técnica de LIFT (Ligadura Interesfintérica del trayecto fistuloso) ha logrado menores tasas de recidiva con casi nulo riesgo de incontinencia según reportes internacionales. Sin embargo, aún no está consolidada como técnica estándar para esta patología. Objetivo: Presentar los resultados (éxito clínico e incontinencia según escala de Wexner) de nuestros pacientes con fístula perianal compleja operados con técnica de LIFT. Materiales y Método: Estudio descriptivo de cohorte prospectiva no aleatoria, con pacientes operados por fístula perianal compleja en el Hospital del Salvador, entre los años 2015 al 2017. Resultados: Se incluyen 22 pacientes operados. En un $77 \%$ se obtiene éxito terapéutico en la primera cirugía y hasta un $90 \%$ con una segunda cirugía más simple. Ninguno de los pacientes modificó su Wexner preoperatorio. Conclusiones: En pacientes con fístula perianal compleja la técnica de $L I F T$ es una alternativa que ofrece igual o mejores tasas de curación clínica con bajo riesgo de incontinencia fecal.

Palabras clave: fístula compleja; LIFT; incontinencia fecal.

\section{Introducción}

La fístula anorrectal representa la fase crónica de un absceso anorrectal y es definida como la persistencia de un trayecto fibroso recubierto por tejido granulatorio entre un orificio primario interno y uno o múltiples orificios secundarios externos en la piel de la región perianal, resultando clínicamente en ensuciamiento, ano húmedo, dolor perianal cíclico y drenaje crónico de material purulento asociado a la formación de abscesos y drenaje espontáneo intermitente $^{1,2}$. Su origen es criptogladular en alrededor de un $90 \%$ de los casos y en el grupo restante es debido a otras afecciones como la enfermedad de 
Crohn, radiación local, cáncer y trauma ${ }^{3}$. La teoría criptoglandular se fundamenta en la exposición constante de carga fecal en el orificio fistuloso interno asociada a la hermeticidad del trayecto, haciendo, finalmente, perpetuar el foco séptico ${ }^{1-3}$.

El objetivo del tratamiento quirúrgico se orienta, básicamente, en lograr la curación del trayecto fistuloso sin alterar la función esfinteriana y evitar en lo posible la recidiva del trayecto ${ }^{3}$. La literatura muestra una variedad de técnicas quirúrgicas para la resolución de las fístulas complejas, aunque algunas logran eficientemente el control de la enfermedad, con una recidiva promedio del $5 \%$, presentan cifras de incontinencia que oscilan entre el $10 \%$ y $57 \%$. En la actualidad, se está buscando la mejor alternativa quirúrgica en fístula anorrectal compleja, lamentablemente la evidencia es baja dado que las series reportadas son muy heterogéneas, de seguimiento variable y breve ${ }^{2}$.

Las técnicas clásicas como la fistulotomía y la técnica con setón o sedal cortante tienen una tasa de recurrencia de hasta un 9\%, provocando en grado variable deterioro de la función anorrectal con las cifras ya descritas ${ }^{4,5}$. La incontinencia fecal es uno de los principales problemas del tratamiento de las fístulas, ya que en ambas técnicas el esfínter anal interno es seccionado ${ }^{2,5}$.

Al considerar los aspectos relacionados a la preservación de la continencia esfinteriana y las tasas variables de recurrencia, se han desarrollado nuevas y variadas técnicas para solventar el problema. Así, han surgido los sellantes biológicos (Fibrin Glue Injection), uso de tapones de distintos materiales, terapias avanzadas relacionadas al uso de Stem Cells y técnicas quirúrgicas novedosas como el LIFT $^{2,5}$.

La ligadura interesfinteriana del trayecto fistuloso (LIFT, del inglés Ligation of Interesphincteric Fistula Tract), considera en su fundamento teórico de base la ligadura y excisión del trayecto interesfinteriano, logrando bloquear la entrada de material fecal en éste, eliminando el sinus séptico criptoglandular crónico $^{4-6}$. La técnica contempla la preservación de ambos esfínteres anales, aspecto que reduce casi por completo el riesgo de incontinencia ${ }^{5,6}$. El primer reporte de esta técnica fue una experiencia tailandesa desarrollada por Rojanasakul et al., en el año 2007, como una modificación a la técnica descrita por Matos et al., en 19937. La descripción de esta técnica se hizo en 18 pacientes con tasas de éxito de un $94 \%$, sin reportar alteraciones en la continencia. Desde entonces, otros autores han publicado sus experiencias con tasas de éxito en relación a curación entre $47 \%$ y $95 \%{ }^{5,6}$, no olvidando el beneficio real respecto a la mantención de la continencia fecal.
Uno de los sesgos más importantes objetivados en las distintas revisiones sistemáticas es la variabilidad detectada en la técnica respecto al LIFT clásico ${ }^{3}$. Se han incorporado, además, técnicas de LIFT híbridas con el uso combinado de biomateriales, que hace aún más complejo el evaluar las potencialidades de la técnica.

El objetivo del presente trabajo, es presentar nuestros resultados en cuanto a eficacia clínica e incontinencia con la técnica de LIFT y potenciar su rol como alternativa de tratamiento para fístulas anorrectales complejas.

\section{Materiales y Método}

Estudio descriptivo de cohorte prospectiva consecutiva, se incluyen 22 pacientes con diagnóstico de fístula perianal compleja, operados con técnica de LIFT entre julio de 2015 y enero de 2017. Para el estudio de la fístula se realiza examen físico e imágenes con resonancia magnética (RM) y/o endosonografía (ES). Se clasifican según Parks ${ }^{8}$. Se incluyen exclusivamente fístulas complejas definidas como transesfinterianas medias y altas, según la proporción de esfínter anal externo comprometido $(>30 \%)$ y fístulas anteriores en la mujer. Se excluyen pacientes con fístulas cuyo origen no fuese criptoglandular; para descartar enfermedad de Crohn se realiza colonoscopia a todos los pacientes. Por historia clínica se descartan otras causas como fístulas actínicas o secundarias a trauma.

A todos los pacientes se les realizó una ligadura interesfinteriana del trayecto fistuloso (LIFT) según la técnica descrita por Rojanasakul modificada, esto es:

\section{Anestesia regional}

Incisión curva en espacio interesfintérico sobre el trayecto de la fístula el que se diseca hasta liberarlo, ligadura y corte de la misma, legrado del trayecto distal y ampliación del orificio externo (OFE).

La modificación incluida fue: legrado del trayecto proximal con gasa e invaginación de los cabos de ligadura con material reabsorbible.

No se realizó el cierre del orificio interno (OFI) (modificación propuesta en otras series) por el riesgo teórico de nuevo absceso.

Los datos demográficos y clínicos se registraron en una base de datos prospectiva, todos los pacientes tienen una evaluación clínica preoperatoria y un seguimiento posoperatorio promedio de 48 semanas. Se define como éxito clínico la ausencia de síntomas o signos de fístula en 3 meses poscirugía. La incontinencia se mide y clasifica según escala de Wexner ${ }^{9}$. 
Los pacientes incluidos en este estudio fueron informados del procedimiento a realizar y sus eventuales complicaciones. Se firmó un documento de consentimiento informado.

\section{Resultados}

Durante el periodo se operan 22 pacientes por fístula perianal compleja con técnica de LIFT, 16 corresponden a hombres y 6 a mujeres. El promedio de edad es de 42 años (i 23-68 años). 18 pacientes corresponden a ASA I y 4 a ASA II. 17 pacientes tienen historia de drenaje de abscesos perianales con anterioridad a la cirugía y el promedio de presentación de síntomas preoperatorio es de 2,2 años (i 0-10 años). En 16 pacientes se decide instalar un setón no cortante previo a la cirugía definitiva. Esto se realiza con el objetivo de manejar el componente infeccioso de la fístula y obtener un trayecto más fibroso. El promedio de tiempo entre setón y LIFT es 60 días. A 6 pacientes se les realiza el LIFT sin la instalación de setón previo. En el estudio preoperatorio, a 20 pacientes se les realiza RM de pelvis con contraste y a 1 endosonografía (Tabla 1). Veinte

Tabla 1. Características de los pacientes

\begin{tabular}{|ll|}
\hline Total & 22 pacientes \\
Edad promedio & 42 años (23-68 años) \\
Hombres-mujeres & $16-6$ \\
Tiempo síntomas promedio & 2,2 años (0-10 años) \\
Drenaje de abscesos previo & 17 \\
Sedal 60 días previos & 16 \\
RNM-endosonografía & $20-2$ \\
\hline
\end{tabular}

pacientes presentan puntaje de Wexner de 0 puntos y 2 de 1 punto en la evaluación preoperatoria. El tiempo promedio de la intervención quirúrgica es de 39,7 minutos. A todos los pacientes se les administra antibiótico profiláctico con ceftriaxona 1 gramo ev. y metronidazol $500 \mathrm{mg}$ ev. Ninguno de los pacientes presenta complicaciones intraoperatorias y a todos se les da el alta a las $24 \mathrm{~h}$ posoperatorias. Al momento del alta a todos los pacientes se les recetó antibióticos por vía oral por 7 días (ciprofloxacino $500 \mathrm{mg}$ cada 12 horas y metronidazol $500 \mathrm{mg}$ cada 8 horas), analgésicos y antiinflamatorios no esteroidales durante 3-5 días.

Se realiza un seguimiento promedio de 48 semanas con un rango (24-96 semanas). El porcentaje de cierre completo fue de un 77\% (17 pacientes). En el seguimiento posoperatorio se objetivó recurrencia de fístula en 5 pacientes $(23 \%)$ con una fístula más simple. A todos se les realizó fistulotomía del trayecto, ahora. Luego de esta segunda intervención se obtuvo un $90 \%$ de éxito clínico en el seguimiento. Ninguno de los 5 casos ha presentado incontinencia. Ningún paciente de los 22 ha modificado su Wexner en el seguimiento (Tabla 2).

\section{Discusión}

Durante mucho tiempo el colgajo de avance de mucosa recto anal fue considerado el gold standard para el tratamiento quirúrgico de fístulas complejas con preservación de la función esfinteriana, mostrando tasas de éxito entre $27 \%-100 \%$, pero con tasas de incontinencia de hasta $35 \%$. Justamente, buscando mejorar estos resultados, el procedimiento de LIFT ha surgido en el último tiempo

Tabla 2. Resumen de resultados

\begin{tabular}{|ll|}
\hline Operados & 22 pacientes \\
\hline Tiempo operatorio promedio & 39,7 minutos \\
\hline Profilaxis ATB & 22 \\
\hline Promedio días hospitalización & 1 \\
\hline Promedio EVA posoperatorio - Alta & $0-1$ \\
\hline Complicaciones precoces & 0 \\
\hline Seguimiento & 48 semanas $(24-96 \mathrm{sem})$ \\
Cierre completo & $77 \%(17 / 22$ pacientes $)$ \\
En 2 etapas* & $90 \%(20 / 22$ pacientes $)$ \\
Recurrencia (todos se trataron con fistulotomía simple) & $23 \%(5$ pacientes con secreción por OFE, downstaging $)$ \\
\hline Recurrencia y sedal previo & $4 / 5$ no tenían sedal** \\
\hline Modificación de Wexner & 0 \\
\hline
\end{tabular}

*Después del primer LIFT se obtuvo cierre primario en el 77\% de los casos, se alcanzó el 90\% de cierre luego de una segunda intervención en los casos pertinentes. $* *$ La recurrencia se presentó en 4 de 5 pacientes, en los cuales no se posicionó setón en la primera fistulotomía. 
como una alternativa atractiva para el tratamiento quirúrgico de la fístula anorrectal compleja. Tiene como principio tratar la fístula y su origen, sin comprometer el aparato esfinteriano. Presenta tasas de cierre definitivo de $47 \%-94 \%$ en las diferentes series publicadas, sin compromiso en la continencia en ninguna de dichas series. Otras ventajas del procedimiento son bajo dolor posoperatorio, rápida reinserción laboral y menor costo económico, frente a otras técnicas que requieren el uso de materiales biológicos.

Revisando la bibliografía, solo existe una publicación nacional respecto al tema, del grupo del Hospital Militar. Este trabajo muestra una experiencia en 9 pacientes de fístulas complejas y fístulas rectovaginales, presentando sólo una recurrencia, con un seguimiento promedio de 33 semanas. Todos evolucionaron sin incontinencia.

El presente reporte de pacientes a los que se les realizó el procedimiento de LIFT demuestra resultados similares a lo reportado en la literatura. $\mathrm{Si}$ bien el número de pacientes es reducido, se acerca a la proporción de pacientes que tienen indicación de un procedimiento complejo y alternativo a la fistulotomía u otras técnicas no quirúrgicas. Cabe destacar que durante el periodo estudiado, solo en el centro principal del estudio (Hospital del Salvador) se operaron 240 fístulas en total, de las cuales solo a $22(9 \%)$ se les realizó el LIFT; lo que pone de manifiesto que la elección de realizar la técnica es muy acotada y, actualmente, no se encuentra dentro de las técnicas preferidas por los cirujanos colorrectales.

En relación a los pacientes del presente estudio, al igual que en las series de pacientes con fístula perianal de origen criptoglandular estos se presentan en edades intermedias, sin mayores comorbilidades por lo que se puede ofrecer de forma segura un procedimiento ambulatorio, con anestesia regional, sin mayores requerimientos de analgésicos y con rápida recuperación tanto funcional como laboral. Al controlar los pacientes durante el posoperatorio ningún paciente sin recidiva tuvo que consultar de urgencia y todos pudieron manejar sus molestias relacionadas a la cirugía con las indicaciones dadas en el momento del alta.

Analizando los resultados, entendiendo como objetivo principal el cierre de la fístula, la presente serie demuestra un cierre de un $77 \%$ solo con el procedimiento de LIFT y si consideramos el cierre en dos etapas luego de una recidiva más simple (interesfinteriana) el porcentaje de cierre se acerca al $90 \%$ (20 de 22 pacientes), con un $100 \%$ de ellos sin alteración de la continencia esfinteriana, lo cual convierte la técnica en una alternativa válida y recomendada en algunos casos específicos de fístula compleja. Al analizar los casos de recidiva (5 pacientes, esto es $23 \%$ del total), 4 de ellos su recidiva fue interesfinteriana, tal como sugiere la literatura como un "downstaging" de un proceso complejo en el cual la recidiva corresponde a la porción más proximal de la fístula, y que en un segundo tiempo se puede solucionar con una fistulotomía simple con buenos resultados y seguridad en la contención esfinteriana. Llama la atención que la mayoría de los pacientes con éxito primario tenían previamente un sedal instalado, lo que sugiere que el drenaje previo y conducción del trayecto podría influir en el éxito de la cicatrización de la fístula, sin embargo, la literatura no es categórica al respecto, dado el número reducido de casos no es posible darle un valor a esta variable. Tampoco podemos concluir que las modificaciones en la técnica influyan en el porcentaje de éxito, pero postulamos que la instalación de un sedal previamente, el curetaje con gasa del trayecto fistuloso, el cierre del orificio interno y la plastía muscular invaginante de los cabos de ligadura podrían influenciar en una mejor cicatrización del cierre disminuyendo los porcentajes de recidiva.

En conclusión, podemos decir que el procedimiento de LIFT, es una alternativa quirúrgica segura, reproducible y con buenos resultados. Presenta una tasa de cierre aceptable y sin alteración de la continencia, por lo que la recomendamos como una buena indicación para fístulas criptoglandulares complejas.

\section{Responsabilidades éticas}

Protección de personas y animales. Los autores declaran que para esta investigación no se han realizado experimentos en seres humanos ni en animales.

Confidencialidad de los datos. Los autores declaran que en este artículo no aparecen datos de pacientes.

Derecho a la privacidad y consentimiento informado. Los autores declaran que en este artículo no aparecen datos de pacientes.

Conflictos de interés: no hay. 


\section{ARTÍCULO ORIGINAL}

\section{Referencias}

1. Steele SR, Kumar R, Feingold DL, Rafferty JL, Buie WD. Standards Practice Task Force of the American Society of Colon and Rectal Surgeons. Practice parameters for the management of perianal abscess and fistula-in-ano. Dis Colon Rectum 2011;54:1465-74.

2. Bannura G. Fístula Anorrectal ¿Son las técnicas nuevas superiores a las clásicas? Rev Chil Cir. 2015;67:43040.

3. Sirany A, Nygaard R, Morken J. The Ligation of the Intersphincteric Fistula Tract Procedure for Anal Fistula: A
Mixed Bag of Results. Dis Colon Rectum 2015;58:604-12.

4. Vergara-Fernández $\mathrm{O}$, Espino-Urbina L. Ligation of intersphincteric fistula tract: What is the evidence in a review? World $\mathrm{J}$ Gastroenterol 2013;19:6805-13.

5. Shanwani AM Nor A, Amri N. Ligation of the Intersphincteric Fistula Tract (LIFT): A sphincter saving technique for fistula in ano. Dis Colon Rectum 2010;53:39-42.

6. Hong K, Kang S, Kalaskar S, Wexner S. Ligation of intersphincteric fistula tract (LIFT) to treat anal fistula: systematic review and meta-analysis. Tech Coloproctol. 2014;18:685-91.

7. Rojanasakul A, Pattanaarun
J, Sahakitrungruang C, Tantiphlachiva $\mathrm{K}$. Total anal sphincter saving technique for fistula in ano; the ligation of intersphincteric fistula tract. J Med Assoc Thai 2007;90:81-6.

8. Barredo C, Leiro F. Abscesos y fístulas perianales. Cir Dig. 2009;III-379:1-11.

Disponible en: www.sacd.org.ar

9. Wainstein C, Quera R, Quijada MI. Incontinencia fecal en el adulto: un desafío permanente. Rev. Med Clin Condes 2013; 24:249-61. Disponible desde: https://www.clinicalascondes.cl/ Dev_CLC/media/Imagenes/PDF\%20 revista $\% 20$ médica/2013/2\%20marzo/8-Dr. Wainstein.pdf 